\title{
Autoreflexivität in Kehlmanns Dramen Geister in Princeton und Die Reise der Verlorenen
}

\section{Autoreflexivity in Kehlmann's Plays Ghosts in Princeton and The Journey of the Lost}

Nikola Mizerová

\begin{abstract}
Abstrakt
Diese Studie schließt eine Forschungslücke in Bezug auf das dramatische Werk von Daniel Kehlmann. Mit seinen insgesamt nur vier Dramen ist der renommierte Romanautor Kehlmann im Bereich des Dramas ein Quereinsteiger und wurde von der Theaterwissenschaft bisher mehr oder weniger übersehen. Seine Dramen fügen sich nahtlos in das Paradigma des gegenwärtigen Theaters ein, für das Poschmann den Begriff kritische Nutzung der dramatischen Form geprägt hat. Der vorliegende Beitrag hat Kehlmanns Dramen Geister in Princeton und Die Reise der Verlorenen als Forschungsgegenstand. Diese Eingrenzung des Themas wird dadurch begründet, dass sich die beiden Dramen von Kehlmanns restlichem dramatischem Werk durch die kritische Nutzung der dramatischen Form mit Rückgriff auf historische Stoffe abheben, und insofern ein organisches Ganzes bilden.
\end{abstract}

\section{Schlüsselwörter}

Daniel Kehlmann, das dramatische Werk Daniel Kehlmanns, deutschsprachiges Theater der Gegenwart, kritische Nutzung der dramatischen Form, Minimalismus

\begin{abstract}
The article investigates the previously unresearched work of Daniel Kehlmann. Kehlmann, a renowned contemporary novelist, has written only four plays, making him a novice in the area of drama. Until now, his dramatic endeavours have been rather overlooked by the researchers in theatre studies; yet, his plays fit in the paradigm of the contemporary theatre, for which G. Poschmann suggests the term 'critical use of dramatic form'. This article focuses on Kehlmann's plays Ghosts in Princeton and The Journey of the Lost. Such thematic focus is explained by the fact that the aforementioned plays differ from the rest of Kehlmann's dramatic work by the critical use of historical subject matter, thus posing as an organic whole.
\end{abstract}

\section{Key words}

Daniel Kehlmann, dramatic work of Daniel Kehlmann, contemporary German-language theatre, critical use of dramatic form, minimalism 
Im Bereich der Dramatik stellt der namhafte Romanautor Daniel Kehlmann einen von der Theaterwissenschaft bisher vernachlässigten Quereinsteiger dar. Noch 2009 behauptete er in seiner kontroversen Festrede zur Eröffnung der Salzburger Festspiele, er habe aus der negativen Erfahrung seines Vaters - des Regisseurs und Schauspielers Michael Kehlmann - gelernt und halte sich absichtlich vom Theater fern (KEHLMANN 2009: 25). Doch das hat sich offenbar inzwischen geändert, wovon Kehlmanns Veröffentlichung Vier Stücke (KEHLMANN 2019) und deren Inszenierungen am Schauspielhaus Graz, am Theater in der Josefstadt in Wien und am Vaudeville Theatre in London ${ }^{1}$ zeugen, die von diesem Autor noch einen wichtigen Beitrag zum deutschsprachigen Drama erhoffen lassen.

Die in dem Band Vier Stücke zusammengestellten Schauspiele Geister in Princeton, Der Mentor, Heiligabend und Die Reise der Verlorenen haben einerseits teil an den typischen Entwicklungstendenzen des gegenwärtigen mitteleuropäischen Dramas, für die bereits in den 90er Jahren Poschmann den Terminus kritische Nutzung der dramatischen Form (POSCHMANN 1997: 97) und Lehmann den Terminus postdramatisches Theater geprägt haben (LEHMANN 2007: 21ff.). Andererseits kann Kehlmann neue Impulse in die gegenwärtige dramatische Produktion einbringen, beispielsweise durch die Einbeziehung von Erkenntnissen aus anderen wissenschaftlichen Fächern, wie sein Drama Geister in Princeton aufzeigt. Dennoch wird sein Name auch weiterhin primär mit seinen Romanen wie Mahlers Zeit (1999), Die Vermessung der Welt (2005) und Tyll (2017) assoziiert, was daran liegen mag, dass er in seiner amerikanischen Wahlheimat vorwiegend in den literarischen Kreisen verkehrt und mit amerikanischen Prosaautoren wie Jonathan Franzen freundschaftliche Beziehungen pflegt. Daneben macht Kehlmann kein Geheimnis aus seiner kritischen Einstellung zum gegenwärtigen deutschsprachigen Regietheater, und seiner Stücke nimmt sich daher nicht jeder Regisseur an. Seine schroffe Ablehnung des Regietheaters formulierte er z.B. in der zornigen Festrede zur Eröffnung der Salzburger Festspiele aus, die später in diesem Beitrag ausführlicher besprochen wird.

Im Folgenden werden Kehlmanns Dramen zunächst paradigmatisch eingeordnet, dann wird auf Kehlmanns poetologische Ausgangspunkte eingegangen, woran Analysen seiner Dramen Geister in Princeton und Die Reise der Verlorenen anschließen. Diese Auswahl lässt sich dadurch begründen, dass sich die beiden Dramen von Kehlmanns restlichem dramatischem Werk durch die von Poschmann postulierte kritische Nutzung der dramatischen Form mit Rückgriff auf historische Stoffe abheben, und insofern ein organisches Ganzes bilden.

1 Beispiele hierfür sind die Inszenierungen am Schauspielhaus Graz und am Theater in der Josefstadt in Wien in den Jahren 2011 (Geister in Princeton), 2012 (Der Mentor), 2017 (Heiligabend) und 2018 (Die Reise der Verlorenen) und am Vaudeville Theatre in London im Jahre 2017 (Der Mentor). 


\section{Paradigmatische Einordnung}

In ihrer Publikation Der nicht mehr dramatische Theatertext aus dem Jahre 1997 versucht Poschmann die unüberschaubare Fülle von Tendenzen im experimentellen Drama seit den 70er Jahren des 20. Jahrhunderts zu charakterisieren. Sie stellt ein neues, nichtdramatisches Funktionsmodell von Theater fest, „das in der Dekonstruktion der Prinzipien von Narration und Figuration und in der konsequenten Delegation der Sinngebung an das Publikum besteht“ (POSCHMANN 1997: 97). Analog zu den vergleichbaren Entwicklungstendenzen in der Prosa seit den 60er Jahren sei nicht mehr die Darstellung einer Fiktion zentral, sondern die Autoreflexivität der Form (POSCHMANN 1997: 97). Ein Theatertext beschreibe somit nicht eine szenisch zu erzählende Geschichte, sondern entwerfe „ein Spiel von z.T. mehrdeutigen szenischen Signifikanten“ (POSCHMANN 1997: 141).

Aus der wirkungsästhetischen Perspektive werde „das Problemfeld aus Wahrnehmung, Verstehen und Bedeutungserzeugung durch (verbalsprachliche oder theatrale) Repräsentation“ in diesen Dramen als Thema in den Vordergrund gerückt (POSCHMANN 1997: 97). „Die Doppelbödigkeit der theatralen Repräsentation arbeitet von Haus aus mit Illusion und Desillusion gleichermaßen“ (POSCHMANN 1997: 136), und das Theater könne daher die moderne Erfahrung, dass es in der unüberschaubaren Welt der Bilder, in der wir leben, für uns keinen Halt gebe, gut vermitteln. Die Wirkung beruhe dann entweder auf der „Erregung von Misstrauen, Beunruhigung, Verunsicherung und Orientierungslosigkeit beim Publikum“, oder aber auf dem „Angebot eines ,Gegenentwurfes‘: die Anregung der Zuschauerphantasie und Eröffnung alternativer Wege der Kognition durch die Ermutigung zum Versuch, Paradoxien und ,Unmögliches' ${ }^{6} \mathrm{zu}$ denken oder das kausallogische Verstehen (Erkennen) durch alogische Erfahrungen (Erleben) zu ersetzen“ (POSCHMANN 1997: 313).

Lehmanns Konzept des postdramatischen Theaters zeigt viele Überlappungen mit dem Ansatz von Poschmann. So verweist Lehmann darauf, dass sich beim postdramatischen Drama seit den 70er Jahren eine wesentliche Reduktion der Handlungslinie vollzieht und dass die szenische Dynamik in den Vordergrund rückt (LEHMANN 2007: 79). Des Weiteren verzichte das postdramatische Drama auf einen hierarchischen Aufbau, der durch eine parataktische Aneinanderreihung von szenischen Bildern ersetzt werde (LEHMANN 2007: 96). Essentiell sei nach Lehmann ein reflexiver Umgang mit dem Dargestellten in Form von Zitaten und Anspielungen (LEHMANN 2007: 110).

Lehmanns Ansatz liefert v.a. durch seine Ausführungen zum „Spiel mit der Dichte der Zeichen“ (LEHMANN 2005: 151) wertvolle Anhaltspunkte für die Analyse von Kehlmanns Dramen. Abgesehen von diesem Merkmal scheint aber der Ansatz von Poschmann durch den zentralen Begriff der Autoreflexivität und die wirkungsästhetische Perspektive für die Analyse von Kehlmanns dramatischem Werk viel aufschlussreicher zu sein, und wird daher in diesem Beitrag als theoretische Basis bevorzugt. Es zeigt sich auch, dass Kehlmann durchaus in der Tradition der kritischen Nutzung der dramatischen Form - so der Terminus von Poschmann (POSCHMANN 1997: 315) - im deutschsprachigen Raum seit den 1970er Jahren steht und dass in seinen Dramen ähnliche 
Verfahren, wie z.B. in den von Poschmann analysierten Dramen von Peter Turrini, Christoph Hein, Elfriede Müller und Friederike Roth u.a., eingesetzt werden. Poschmanns Terminus kritische Nutzung der dramatischen Form ist auf Kehlmanns Dramen ohne Weiteres beziehbar. Im Unterschied zu dem radikalen Metadrama, das ausschließlich autoreflexiv ist, stellt Poschmann den Begriff der kritischen Nutzung der dramatischen Form auf, um auch diejenigen Dramen zu erfassen, die zwar eine narrative Handlungslinie beibehalten, diese jedoch autoreflexiv, kritisch nutzen, um die entsprechende, oben beschriebene Wirkung zu erzielen.

Häufig geht Poschmann zufolge diese kritische Nutzung entweder mit einem Rückgriff auf historische Stoffe oder mit den Mitteln des Absurden und Phantastischen einher. Im Falle des Rückgriffs auf historische Stoffe erzähle zwar das Drama eine historische Geschichte, zugleich werde jedoch - wie z.B. in Christoph Heins Dramen Passage und Ritter der Tafelrunde - mithilfe von vielen im Text eingebauten Unstimmigkeiten „eine Alternative zur kausal-logischen Rationalität“ praktiziert (POSCHMANN 1997: 78). Es komme häufig zur „Vervielfältigung der Fiktionsebenen“, die in die Unmöglichkeit mündet, diese klar zu hierarchisieren. Diese überreale Fiktionsebene verselbstständige sich und es entstehe ein „Schwebezustand zwischen Wirklichkeit und Traum“ (POSCHMANN 1997: 132). Die Hierarchisierung der beiden Ebenen (als dargestellte Realität einerseits und Wahrnehmung einer Figur andererseits) werde als fragwürdig präsentiert (POSCHMANN 1997: 103), wodurch verschiedene Lesarten offengelassen werden (POSCHMANN 1997: 103). Sie könne sich auch „als Außerkraftsetzen der Gesetze von Raum und Zeit in einer Dramaturgie der Traumlogik [...] oder als Angebot mehrerer verschiedener, sich nach klassischer Logik [...] wechselseitig ausschließender Geschichten" verwirklichen (POSCHMANN 1997: 103). Durch die Verunsicherung der ZuschauerInnen mit Hilfe paradoxer Effekte der Selbstbezüglichkeit, wie z.B. „durch die Eigengesetzlichkeit des Bühnengeschehens, das sich mit den Mitteln der klassischen Logik nicht in eine Geschichte rückübersetzen lässt, da die Gesetze von Raum, Zeit oder Kausallogik gebrochen und so Paradoxien produziert werden“ (POSCHMANN 1997: 133), werde in diesen Dramen die „Unzuverlässigkeit und Subjektivität kognitiver Prozesse, Wirkungsmuster des Unbewussten und Unterbewussten“ zum Hauptthema (POSCHMANN 1997: 116). Die ZuschauerInnen müssen also die Erfahrung machen, dass die Wahrheit im Theater nur eine Illusion ist (POSCHMANN 1997: 315).

Wie im Folgenden bewiesen wird, sind auch Kehlmanns Dramen Geister in Princeton und Die Reise der Verlorenen stark autoreflexiv angelegt und zielen darauf ab, die $\mathrm{Zu}$ schauerInnen zu verunsichern und sie die Unzulänglichkeit der Vernunft und Wahrnehmung hautnah erleben zu lassen. Ähnlich wie die oben genannten Autoren erreicht dies Kehlmann in den beiden Dramen, indem er eine historische Geschichte autoreflexiv gestaltet. Die Autoreflexivität wird bei ihm entweder erreicht, indem eine historische Geschichte mit einer zweiten Fiktionsebene gekoppelt wird und zugleich Gesetze der Logik und Kausalität aus den Angeln gehoben werden, wie in seinem Drama Geister in Princeton, oder indem eine historische Geschichte durch traditionelle Verfremdungseffekte unterminiert wird, wie im Drama Die Reise der Verlorenen. 


\section{Kehlmanns poetologische Ausgangspunkte}

Der Tatsache entsprechend, dass sich Kehlmann primär als Romanautor profiliert, sind poetologische Aussagen zum Drama in seinem Werk eher spärlich. Immerhin kann sich eine theaterwissenschaftliche Betrachtung auf seine bereits erwähnte Festrede zur Eröffnung der Salzburger Festspiele (KEHLMANN 2009: 25) und auf seine Poetik-Vorlesungen (KEHLMANN 2010, 2015, 2016) stützen. Von diesen Quellen ausgehend, wird im Folgenden ein Konzept erläutert, das für Kehlmanns dramatisches Werk ausschlaggebend ist, und zwar der Minimalismus.

Wie aus seiner Salzburger Rede herauszulesen ist, strebt Kehlmann im Drama nach einem ausgeprägten Minimalismus (KEHLMANN 2009: 25). Nach eigenen Worten wurde er in dieser Hinsicht von seinem Vater, dem Theaterregisseur und Schauspieler Michael Kehlmann, beeinflusst (KEHLMANN 2009: 25). In der Salzburger Rede, die als ein Versuch wahrzunehmen ist, seinen Vater nachträglich zu ehren und zu rehabilitieren ${ }^{2}$, wendet sich Kehlmann gegen das große, Effekthascherei betreibende Ausstattungstheater. Er äußert sich in diesem Zusammenhang auch sehr kritisch über hochsubventionierte Spektakelstücke, die es mit ihren immergleichen, aufwendigen Bühnenbildern und ihrer theatralischen Künstlichkeit („ständig Videowände und Spaghettiessen, warum [... ist] immer irgendwer mit irgendwas beschmiert, wozu all das Gezucke und routiniert hysterische Geschrei?" [KEHLMANN 2009: 25]) auf den deutschsprachigen Philister abgesehen hätten. Als Alternative dazu plädiert Daniel Kehlmann für einen Minimalismus, als dessen Vorbilder und Vertreter er die Dramatiker Karl Kraus ${ }^{3}$ und Samuel Beckett ${ }^{4}$ und den Regisseur Peter Brook nennt (KEHLMANN 2009: 25).

Zwischen Brook und Kehlmann besteht eine Reihe von Berührungspunkten. Neben dem Respekt vor Beckett ist es auch die Hochachtung vor Shakespeare und dem elisabethanischen Theater, die beiden als Inspirationsquellen und Vorbilder des modernen Theaters dienen. In seinen Poetik-Vorlesungen Kommt, Geister bezieht sich Kehlmann wiederholt auf Shakespeares Dramen (KEHLMANN 2015) und äußert sich in diesem

2 In den letzten Jahrzehnten seines Lebens durfte Michael Kehlmann nicht als Regisseur arbeiten, da er - soweit Daniel Kehlmann - als altmodisch galt (KEHLMANN 2009: 25).

3 Kehlmann versteht Karl Kraus als einen Gegner des Regietheaters und einen Anhänger des Minimalismus, siehe sein Kommentar: „Kraus war in keiner Weise ein Anhänger des großen Ausstattungstheaters, er trat für äußerste Reduktion ein; was ihm vorschwebte, war näher bei dem Minimalismus eines Peter Brook als bei Max Reinhardt“ (KEHLMANN 2009: 25). Dabei beruft sich Kehlmann v.a. auf Kraus“ Aufsatz Mein Vorurteil gegen Piscator aus dem Jahre 1926, in dem Kraus Piscators „aktualisierte“ Aufführung von Schillers Räubern kritisiert und feststellt: „'Aktuell ‘...] ist die Überwindung des Zeitwiderstands, die Wegräumung des Überzugs, den das Geräusch des Lebens dem Gehör und der Sprache angetan hat. Für aktuell aber halten die Zutreiber der Zeit den Triumph des Geräusches über das Gedicht, die Entstellung seiner Geistigkeit durch ein psychologisches Motiv, das der Journalbildung [...] erschlossen ist“" (KEHLMANN 2009: 25).

4 Kehlmann nennt Beckett explizit einen Minimalisten und einen Gegner des Regietheaters: „Ein anderer Minimalist, Samuel Beckett, verbot regelmäßig Aufführungen seiner Werke, die er als entstellend empfand und die von seinen akribischen Regieanweisungen abwichen - möchte man ihn darum rückständig nennen?" (KEHLMANN 2009: 25) Übrigens sei noch ergänzt, dass Beckett von Poschmann ebenso wie von Lehmann als typischer Vertreter des nichtdramatischen Funktionsmodells vom Theater und des postdramatischen Theaters bezeichnet wird (POSCHMANN 1997; LEHMANN 2007). 
Zusammenhang auch zu Brooks viel gerühmter Shakespeare-Inszenierung des Midsummer Night's Dream:

Peter Brook bricht 1970 in Stratford kurzerhand mit allen Traditionen der Lieblichkeit. Er lässt die Nacht zum Tag werden, taucht die Szenerie in weißes Licht und macht das Stück zu einer Zirkusvorstellung mit Trapez, Stelzengängern und Jongleuren: keine Aktualisierung, sondern Abstraktion, die Übertragung des Dramas in den durch Brook sprichwörtlich gewordenen leeren Raum [...]. Brook ist ein Zen-Buddhist, der an die Unwiederholbarkeit des theatralischen Moments glaubt. (KEHLMANN 2015: 89)

Vor dem Hintergrund von Kehlmanns Salzburger Rede lässt sich aus dieser Einschätzung schlussfolgern, dass es v.a. die Abkehr von jeder Gefälligkeit, das Konzept des leeren Raums, die Abstraktion und die Unwiederholbarkeit des Theatererlebnisses sind, die Brooks und Kehlmanns Theaterpoetik miteinander verbinden.

Brooks Inszenierung des Midsummer Night's Dream mit seinem Bühnenbild in Form der ikonischen „White Box“ ist ein mittlerweile berühmtes Beispiel für sein Konzept des leeren Raums (BROOK 1983). Dies formuliert Brook für das moderne Theater u.a. in Auseinandersetzung mit dem elisabethinischen Theater, dessen zeitlosen Minimalismus er preist und das ihm als vorbildlich für das moderne Drama gilt:

Die elisabethanische Bühne [...] war ein neutrales offenes Podium - nichts als eine Fläche mit ein paar Türen - und gab dadurch dem Dramatiker die Möglichkeit, den Zuschauer mühelos durch eine unbegrenzte Folge von Illusionen zu jagen, die, wenn er es wollte, die ganze physische Welt beinhalteten. (BROOK 1983: 113)

Während Lehmann den besagten Brook für einen der ganz großen Namen hält, die untrennbar mit dem postdramatischen Theater verbunden sind (LEHMANN 2007: 20), wird er bei Poschmann völlig ausgeklammert, da sich die Autorin ausschließlich auf das deutschsprachige Theater beschränkt (POSCHMANN 1997). Lehmann erwähnt Brooks Inszenierungen u.a. als ein Beispiel für das „Spiel mit der Dichte der Zeichen“ (LEHMANN 2005: 151). Die Verstöße gegen die gewohnte Dichte theatraler Zeichen, die sich in der Dialektik der Fülle und Leere vollziehen, werden von Lehmann als Mittel dazu angegeben, die Autoreflexivität des Theaters zu thematisieren. Als Gegenpol zur Überfüllung durch theatrale Zeichen (beim Regisseur Jürgen Kruse) sieht Lehmann die Vorliebe für die leere Szene (bei Bertolt Brecht, beim Regisseur Peter Brook) oder fürs Schweigen (bei Peter Handke, Samuel Beckett, John Cage).

Eine ähnliche Vorliebe für eine minimalistisch leere Szene zeigen auch Kehlmanns Dramen. Als Beispiel ist hier Kehlmanns Einakter Heiligabend heranzuziehen, der sich auf einen Dialog von zwei Schauspielern und das folgende Bühnenbild beschränkt: „Ein spärlich möblierter Raum: Stühle, ein Tisch mit Papierstapeln sowie Thermoskanne und Pappbechern, eine Uhr" (KEHLMANN 2019: 149). Im Laufe der Inszenierung kommt übrigens noch ein Telefon dazu. Analoges gilt für alle seine Dramen ohne Unterschied. 
Im Einklang mit seiner Salzburger Rede verzichtet Kehlmann völlig auf große Effekte und Gesten. Die tragende Rolle kommt dem gesprochenen Text zu. Dadurch erinnern Kehlmanns Dramen z.B. an Christoph Hein, den übrigens Poschmann einen Vertreter der kritischen Nutzung der dramatischen Form nennt, was noch später weiter ausgeführt wird. Ebenso wie bei Christoph Hein handelt es sich bei Kehlmanns Dramen um einen „konventionellen Typus des Konversationsstücks“, um einen „Versuch, in der Nussschale zu sein“ (POSCHMANN 1997: 94). Ebenso wie bei Hein geschehen auch bei Kehlmann alle Handlungen, die mit großen Effekten verbunden wären und die $\mathrm{Zu}$ schauerInnen ablenken würden, wie Krieg (in Heins Passage und Kehlmanns Geister in Princeton), außerhalb der Bühne und werden in den Stücken nur besprochen.

\section{KehImanns Drama Geister in Princeton}

Kehlmanns biographisches Drama über den Mathematiker Kurt Gödel, Geister in Princeton, ist mehr als eine literarische Bearbeitung der bewegten Lebensgeschichte eines lebensuntüchtigen Genies. Geister in Princeton haben den ZuschauerInnen viel mehr zu bieten als biographische Daten, indem sie zugleich Gödels wissenschaftliches Konzept miteinbeziehen und kreativ umgestalten. Kehlmann lässt in das Drama v.a. Gödels Beweis einfließen, „dass unter sehr speziellen Umständen - in einer Welt rotierender Galaxienhaufen nämlich, in der die Raumzeit sich gewissermaßen in sich selbst zurückkrümmt - Zeitreisen in die Vergangenheit möglich sind“" (KEHLMANN 2015: 155). Diese wissenschaftliche Entdeckung verwertet Kehlmann, um eine magisch anmutende Welt, eine zweite Fiktionsebene zu schaffen, die in die alltägliche, biographische Realität eingreift und sie entfremdet.

Das Schauspiel beginnt mit Gödels Bestattung, und die nachfolgenden Ereignisse, die Gödels biographische Daten getreu nachzeichnen, können damit als Rückblende gedeutet werden. Die einzelnen Szenen geleiten die ZuschauerInnen durch Gödels Leben. Er wächst in einer wohlhabenden deutschböhmischen Familie in Brünn auf, wandert nach dem Zerfall der Habsburger Monarchie nach Wien aus, da er die neu gegründete Tschechoslowakei nicht für seine Heimat hält. Trotz seines jungen Alters wird er Mitglied des Wiener Kreises. 1933 habilitiert er sich, doch als Privatdozent hält er Vorlesungen praktisch ohne Entgelt. Als Ausweg aus der finanziell trostlosen Lage bieten sich - insgesamt drei - längere Arbeitsaufenthalte am Institute of Advanced Studies an der Princeton-Universität, das als eine Art Forschungsparadies bekannt ist. Nach dem „Anschluss“ Österreichs verliert Gödel seine Stelle an der Universität, wird paradoxerweise als Vertreter einer „stark verjudeten Mathematik“ (SIGMUND et al. 2016: 72) für einen Juden gehalten, und dadurch erneut heimatlos. Seine Flucht über die Sowjetunion und Japan nimmt schließlich in den USA ein glückliches Ende, wo er dank seiner früheren Kontakte eine feste Anstellung an der Princeton-University findet. 1953 wird er an dieser Stelle zum Professor für Mathematik ernannt. Mit Einstein ist Gödel in seinen amerikanischen Jahren eng befreundet, sie treffen sich tagtäglich. Als Professor in Princeton erlebt Gödel mit, wie seine Kollegen (z. B. John von Neumann) an der 
Atombombe arbeiten, und später in der McCarthy-Ära (z. B. Robert Oppenheimer) politisch verfolgt werden. Lebenslang wird Gödel von psychischen Krisen heimgesucht. An seinem Lebensabend verschlimmert sich seine Paranoia, die er bereits in den 30er Jahren entwickelte. Er nimmt viele Medikamente zu sich. Er ist überzeugt, Geister zu sehen und ist von der Idee besessen, dass man ihn vergiften will. Nachdem seine Frau Adele, die ihn zeitlebens pflegt, selber im Krankenhaus landet, verhungert er, jegliche Nahrung verweigernd.

Diese Stationen aus Gödels Leben werden im Drama in der chronologischen Reihenordnung szenisch dargestellt. Trotz dieser Haupthandlungslinie ist es für die ZuschauerInnen durchaus nicht so problemlos, sich einen genauen Überblick zu verschaffen. Die Handlung wird nämlich bewusst als ein „Fiebertraum“ - soweit die Formulierung der Figur Einsteins im Drama - inszeniert. Auf der Bühne scheint sich Gödels Konzept der Zeit zu verkörpern und mit den biographischen Daten zu einer neuen, verunsicherten Wirklichkeit zu verschwimmen.

Gödels Entdeckung in Anlehnung an Einstein, „dass unter sehr speziellen Umständen [...] Zeitreisen in die Vergangenheit möglich sind" (KEHLMANN 2015: 156f.), stellt die traditionelle, lineare Zeitauffassung in Frage. Es wird zwar die grundlegende chronologische Anordnung der biographischen Szenen beibehalten. Doch im Sinne des Konzepts möglicher Zeitreisen begegnet auf der Bühne die Figur „Gödel“ wiederholt den Figuren „Gödel als Kind“ und „Gödels Alter Ego“ und interagiert mit ihnen. Ähnlich treten im Laufe des Dramas kontinuierlich Gödels bereits verstorbene Freunde und Bekannte auf und führen Gespräche mit ihm. In Gödels Weltbild sind seine jüngeren und älteren Ich sowie seine toten Freunde und Bekannten anwesend, da er logisch nachgewiesen und mathematisch berechnet hat, dass es diese Möglichkeit durchaus gebe. Doch sind sie dadurch auch „real“ präsent? Und ist das, was physikalisch möglich ist, wirklich auch umsetzbar?

Durch das Auftreten der vervielfältigten Ich und der bereits Verstorbenen entsteht jedenfalls das Bild einer Zeit, die zyklisch abläuft. Die unheimlichen Konsequenzen werden von „Gödels Alter Ego“ in der zweiten Szene ausformuliert, wo es zu der Figur „Gödel“ sagt: „und alles, was du versäumt hast, bleibt versäumt in Ewigkeit und wird wieder versäumt, und was du getan hast, tust du von neuem. Jedem seine eigene Hölle. Das ist deine" (KEHLMANN 2019: 20). Die zyklische Zeitauffassung wird dann im Drama nicht nur durch ständige Begegnungen der vervielfachten Figuren von Gödel und der Toten verwirklicht, sondern auch durch den zyklischen Aufbau des Dramas. Und die ZuschauerInnen müssen im Laufe des Stücks anerkennen, dass es sich strenggenommen um keine „einfache“ Retrospektive nach dem Tod des Protagonisten handelt, sondern dass die Zeit „tatsächlich“ im Kreis läuft, dass sich Gödels Leben traumhaft zyklisch zu wiederholen scheint. Das kann mit Hilfe der vorletzten Szene sehr gut illustriert werden. Hier reden alle drei Gödels miteinander darüber, wer von ihnen eigentlich träumt, wer „real“ ist und wie die Zeit läuft: 
Gödel als Kind: Ich träume das, nicht wahr?

Gödel: Diesmal bin ich es wohl, der träumt.

Gödels Alter Ego: Macht es einen Unterschied?

Gödel zum Kind: Wenn du es bist, wird alles noch geschehen.

Wenn ich es bin, ist es schon vorbei.

Gödels Alter Ego: In jedem Fall ist es schon passiert, in jedem Fall passiert es wieder.

Der Zug fährt im Kreis. (KEHLMANN 2019: 84)

Man könnte natürlich vermuten, dass die „Geister“ und die zyklische Zeitauffassung nur Ausgeburten des kranken Gemüts Gödels seien. Dieser Annahme widerspricht jedoch das Drama. In der hier dargestellten Welt, die durch Verschmelzung Gödels realer Biographie und seiner Ideenwelt entsteht, ist nämlich die Hierarchisierung beider Fiktionsebenen nicht klar und es scheint die Logik „sowohl - als auch“ zu gelten.

Eine Schwarz-Weiß-Malerei in dem Sinne, Gödel sei am Lebensabend verrückt geworden und das Drama reflektiere nur sein krankes Gemüt, vermeidet Kehlmann völlig. Wie er es in einer Poetik-Vorlesung auf den Punkt bringt, sei Gödel genial und paranoid zugleich gewesen, man könne sich also nicht für eine von den beiden Alternativen entscheiden: „Man kann nicht behaupten, dass Gödel zuerst ein großer Logiker ist und später den Verstand verliert. Er behält den Verstand bis zum letzten Tag, aber er ist auch paranoid und sieht Gespenster, und die Vernunft kommt ihm nicht zu Hilfe [...]“ (KEHLMANN 2015: 156).

Dem Ansatz von Poschmann entsprechend, ist Kehlmanns Drama Geister in Princeton ein anschauliches Beispiel für die kritische Nutzung der dramatischen Form. Die narrative, biographische Linie dient dazu, ein Paradoxon zu kreieren: der große Logiker und Mathematiker des 20. Jahrhunderts kann sich nicht auf die eigene Vernunft verlassen. Sein Konzept der Zeit verselbstständigt sich und bricht die uns vertrauten Gesetze von Zeit und Kausallogik. Die narrative Linie dient also nicht primär dazu, eine Geschichte zu erzählen, sondern die ZuschauerInnen zu verwirren, zu beunruhigen. In diesem Stück wird eine „enge Verknüpfung der Bereiche Theater und Erkenntnis“ vollzogen (POSCHMANN 1997: 116). Für die ZuschauerInnen ist das Drama ein dunkles Labyrinth, das sie die fatale Schwäche der menschlichen Vernunft und Logik hautnah erleben lässt. Und dies ist schließlich auch die Hauptaussage des Schauspiels, die über das Biographische und Zitathafte hinausgeht: Die Vernunft ist brüchig. Die Gesetze der Logik helfen uns in der Auseinandersetzung mit der Welt zwar weiter, aber sind sie wirklich verlässlich? Mit Gödels Alter Ego im Drama Geister in Princeton gesprochen: „Die Welt ist vernünftig, aber sie enthält Fehler. Sie hat schadhafte Seiten, Risse. Ist die Vernunft konsistent? Wir können es nicht wissen. Vielleicht ist die Vernunft ein Alptraum. Vielleicht sind wir verrückt, wenn wir meinen, am klarsten zu sein“ (KEHLMANN 2019: 20). 


\section{Kehlmanns Drama Die Reise der Verlorenen}

Ebenfalls Kehlmanns Drama Die Reise der Verlorenen soll an dieser Stelle als Beispiel für die von Poschmann definierte kritische Nutzung der dramatischen Form dienen. Doch im Unterschied zum Text Geister in Princeton verfährt Kehlmann in diesem Drama andersartig. Die Reise der Verlorenen basiert zwar gleichfalls auf einer historischen Geschichte, ebenso wie Geister in Princeton, diesmal wird jedoch keine zweite Fiktionsebene miteinbezogen, und die Autoreflexivität des Stücks wird primär durch Verfremdungseffekte erreicht.

Das Drama referiert auf ein unmoralisches Unterfangen, bei dem die Nazis 1939 das Schiff St. Louis mit knapp ein Tausend Passagieren jüdischer Herkunft nach Kuba abfertigen ließen. Es handelte sich um ein nazistisches Täuschungsmanöver, das bei der Weltöffentlichkeit den Eindruck erwecken sollte, die Juden hätten die Möglichkeit, aus Deutschland frei auszureisen. In Wirklichkeit war jedoch von Anfang an augenscheinlich, dass die Flüchtlinge in Kuba nicht aufgenommen werden und zur Rückkehr nach Deutschland verdammt waren. Das Schiff durfte in Kuba nicht mal anlegen, und die Aufnahme der Flüchtlinge wurde vom kubanischen Präsidenten als derartig gefährliches Präzedens gedeutet, das sich das arme Land nicht einmal hätte leisten können. So gelang es den Nazis geschickt, die Verantwortung und die eventuelle Schuld auf die anderen Länder abzuwälzen, da die St. Louis auch von den USA abgelehnt wurde. Das Schiff musste nun seine Rückreise nach Deutschland antreten. Die Stimmung auf dem Schiff kippte natürlich und manche Passagiere drohten mit Massensuizid.

All diese Begebenheiten werden im Drama erfasst. Fokussiert wird das moralische Dilemma des Kapitäns Gustav Schröder. Soll er nur seine Pflicht erfüllen, oder soll er versuchen den Flüchtlingen Hilfe zu leisten? Er entscheidet sich schließlich dazu, das Schiff vor Sussex auf Grund laufen zu lassen, da dann alle - wenn der Plan aufgehen sollte - von der englischen Küstenwache gerettet werden. Natürlich muss er dabei in Kauf nehmen, dass sein Handeln als Versagen des Kapitäns beurteilt und dass er anschließend vor Gericht gestellt wird. Glücklicherweise gelingt es den jüdischen Organisationen noch vor seiner verzweifelten Tat, eine Einigung mit ausgewählten europäischen Ländern zu erzielen. Das Schiff darf nun in Holland anlegen und die Flüchtlinge werden auf Holland selbst, Frankreich sowie England verteilt.

Soweit die narrative Linie des Stücks. Von der ersten Szene an ist jedoch das Drama betont autoreflexiv. So beginnt es mit einem klassischen Verfremdungseffekt - einer Anrede, in welcher der Antiheld, der nazistische Agent Schiendick, aus der Rolle tritt und sich an das Publikum wendet:

Wir schreiben das Jahr 1939. Und sofort schätzen Sie sich glücklich, nicht wahr, dass Sie diese Zeit nicht erlebt haben. [...] Und Sie, begnadet mit später Geburt, denken vielleicht gerade: „Wer weiß, wie ich gehandelt hätte?“ [...] Diese Geschichte ist wahr. Uns alle hat es gegeben. [...] Und alle sind wir jetzt tot, egal, auf welcher Seite wir standen. Nur ist es eben nicht egal.

Es ist nie egal, auf welcher Seite einer steht. (KEHLMANN 2019: 200) 
Damit wird bereits in der ersten Szene die Botschaft des Stücks erwähnt: Es ist nicht egal, auf welcher Seite man steht. Die Geschichte des Schiffs mit Flüchtlingen wird konsequent als schwarz-weiß präsentiert, wodurch sie ein universelles moralisches Handlungsmuster darstellt. Die im Drama kontinuierlich verwendeten Verfremdungseffekte dienen dazu, bei den ZuschauerInnen einen Abstand zu der Geschichte herzustellen, und dank dessen erweist sich die Geschichte als recht gut überschaubar. Dabei werden die traditionellen Verfremdungseffekte eingesetzt, wie das Unterbrechen einer Handlung durch Kommentare oder Zeitsprünge, das Treten von Figuren aus ihrer Rolle, Sprechen von Figuren zum Publikum, Zitieren von Quellen. Auch der scheinbar glückliche Ausgang der Geschichte, den man zu den Mitteln der Illusionsbildung zählen könnte, wird unterminiert, indem Kehlmann in der vorletzten Szene ausgewählte Passagiere ihren weiteren Lebensweg vorwegnehmen lässt:

BABETTE SPANIER: [...] Als die Deutschen kamen, waren wir schon im Lager. Sie mussten Westerbork nur übernehmen. Jede Woche kamen neue Züge mit Verhafteten. Jede Woche fuhren Züge nach Polen ab.

POZNER: In einem davon war ich. Ich wurde in Auschwitz vergast. Das wissen Sie, ich habe es Ihnen ja schon gesagt. Mein Tagebuch hat überdauert, nur deshalb weiß man, dass es mich gegeben hat, Aaron Pozner, einen armen Hebräischlehrer. Was aus meiner Familie wurde, ist nicht bekannt. (KEHLMANN 2019: 282)

Wie bereits gesagt, geht es in dem Drama weniger um die bewegte Geschichte des Schiffes St. Louis und dessen jüdischer Passagiere, als eher darum, wie die ZuschauerInnen ähnliche, ihnen präsentierte Bilder kognitiv bearbeiten. Bereits aus der Wahl des Stoffes wird ersichtlich, dass im Drama viel mehr die mediale Vervielfältigung dieser Geschichte fokussiert wird als die konkrete Geschichte an sich. Das Stück geht nämlich sowohl von der wahren Geschichte des Schiffes St. Louis aus, als auch von deren Bearbeitung im Buch The Voyage of the Damned (1974) von Gordon Thomas und Max Morgan-Witts sowievon dem gleichnamigen Film (1976) vom Regisseur Stuart Rosenberg.

Und es ist sicherlich kein Zufall, dass Kehlmann gerade das Motiv des abgelehnten Schiffes mit Flüchtlingen aufgreift. Es handelt sich nämlich um ähnliche Bilder, die uns gegenwärtig in den Medien im Zusammenhang mit der Flüchtlingskrise geboten werden.

Durch eine Geschichte, die durch Verfremdungseffekte unterminiert wird, um bei den ZuschauerInnen einen Abstand und eine Verunsicherung hinsichtlich des eigenen moralischen Handelns zu bewirken, erinnert Kehlmanns Drama sehr stark an Bertolt Brecht, der übrigens sowohl von Poschmann als auch von Lehmann für einen frühen Vertreter des neuen nichtdramatischen Funktionsmodells von Theater und des postdramatischen Theaters erwähnt wird. Dabei ist auch die unübersehbare Anlehnung an Brecht als ein Zitat, also ein autoreflexives Mittel, zu deuten. 


\section{Fazit}

Zusammenfassend kann festgestellt werden, dass auf beide an dieser Stelle analysierten Dramen Poschmanns Terminus kritische Nutzung dramatischer Form mit Rückgriff auf historische Stoffe anwendbar ist. Geister in Princeton und Die Reise der Verlorenen basieren zwar auf einer historischen Geschichte, sind aber in hohem Maße autoreflexiv und zielen auf die Verunsicherung der ZuschauerInnen ab.

In Geister in Princeton verkörpert sich auf der Bühne eine zweite, abstrakte bis fantastisch anmutende Fiktionsebene mit einer anderen als der linearen Zeitauffassung, die den biographisch fundierten Alltag unsicher erscheinen lässt. Die Hierarchisierung der beiden Fiktionsebenen wird dabei als fragwürdig präsentiert. Das Drama lässt dadurch die ZuschauerInnen die Grenzen der Vernunft und Logik hautnah erleben, indem es sie dazu bringt, vergeblich über die dargestellten Paradoxa der Zeit zu grübeln. Irritierend ist dabei, dass auf die zweite, abstrakte Fiktionsebene als auf eine wissenschaftlich nachgewiesene Entdeckung referiert wird, und dass sie dennoch im scharfen Widerspruch zu unserem alltäglichen Erfahrungshorizont steht und sich fantastisch anmutet.

In Die Reise der Verlorenen wird eine historische Geschichte durch ihre mediale Vervielfältigung und durch die klassischen Verfremdungseffekte unterminiert, wodurch die Illusionsbildung kontinuierlich verhindert wird. Der so hervorgerufene Abstand zum Erzählten soll bei den ZuschauerInnen die Reflexion einer in der Vergangenheit liegenden, und dadurch überschaubaren Geschichte bewirken, die als ein schwarz-weiBes moralisches Handlungsmuster präsentiert wird. Die Verunsicherung der ZuschauerInnen wird gleich in der ersten Szene bewirkt, indem an sie eine Frage gerichtet wird, wie sie selbst in einer ähnlichen Situation handeln würden. Die Parallele zur gegenwärtigen Flüchtlingskrise ist für die ZuschauerInnen unverkennbar. Sie werden dazu gebracht, ihre Einstellung zur Flüchtlingskrise zu revidieren, indem ihnen die eindeutige Botschaft präsentiert wird: Es ist unmoralisch, Flüchtlinge abzulehnen und es nicht egal, auf welcher Seite man steht. Durch seine erzieherische Wirkung und durch den Einsatz von Verfremdungseffekten lehnt sich das Stück sehr deutlich an die Lehrstücke von Bertolt Brecht an und nutzt zugleich diese Quelle autoreflexiv als Zitat.

\section{Bibliografie}

BROOK, Peter. 1983. Der leere Raum. Übersetzt von Walter Hasenclever. Berlin: Alexander Verlag, 1983.

KEHLMANN, Daniel. 2009. Wo heute Lärm ist, war einst Magie. Frankfurter Allgemeine Zeitung (27. 7. 2009). [letzter Zugriff 15. 10. 2020]. Online verfügbar unter https://www.faz.net/aktuell/feuilleton/debatten/theater-wo-heute-laerm-ist-war-einst-magie-1623520.html.

KEHLMANN, Daniel. 2010. Wo ist Carlos Montúfar? Über Bücher. 3. Auflage. Reinbek bei Hamburg: Rowohlt Taschenbuch Verlag, 2010. 
KEHLMANN, Daniel. 2015. Kommt, Geister. Frankfurter Vorlesungen. 2. Auflage. Reinbek bei Hamburg: Rowohlt Verlag, 2015.

KEHLMANN, Daniel. 2016. Die sehr ernsten Scherze. Poetikvorlesungen. 5. Auflage. Göttingen: Wallstein, 2016.

KEHLMANN, Daniel. 2019. Vier Stücke. Reinbek bei Hamburg: Rowohlt Verlag, 2019.

LEHMANN, Hans-Thies. 2005. Postdramatisches Theater. 3. Auflage. Frankfurt a. M.: Verlag der Autoren, 2005.

LEHMANN, Hans-Thies. 2007. Postdramatické divadlo [Postdramatic Theatre]. Překl. Anna Grusková a Elena Diamantová. Bratislava: Divadelný ústav, 2007.

POSCHMANN, Gerda. 1997. Der nicht mehr dramatische Theatertext. Aktuelle Bühnenstücke und ihre dramaturgische Analyse. Tübingen: Max Niemeyer Verlag, 1997.

SIGMUND, Karl, John DAWSON und Kurt MÜHLBERGER. 2006. Kurt Gödel. Das Album. Wiesbaden: Vieweg, 2006. 


\section{Mgr. Nikola Mizerová, Ph.D.}

Technická univerzita $\vee$ Liberci

Fakulta př́rodovědně-humanitní a pedagogická

Katedra německého jazyka

Komenského 314/2, 46001 Liberec, Česká republika

nikola.mizerova@tul.cz

Nikola Mizerová, wissenschaftliche Assistentin am Institut für deutsche Sprache an der Technischen Universität in Liberec. Forschungsschwerpunkte: die Gattung der Groteske in der deutschsprachigen Literatur aus den Böhmischen Ländern, Daniel Kehlmann, literarische Reflexion des Gödelschen Unvollständigkeitssatzes, Literatur und Naturwissenschaften.

Nikola Mizerová works as an assistant professor at the Department of German Language, Technical University of Liberec. In her research, she focuses on the contemporary Germanlanguage literature, Czech and Moravian literature written in German, the genre of grotesque comedy, and literary reflections of the work of the mathematician Kurt Gödel. 